\title{
HuR induces inflammatory responses in HUVECs and murine sepsis via binding to HMGB1
}

\author{
JIAN WANG ${ }^{1}$, LING ZHAO $^{2}$, YONG LI $^{1}$, SHUNYI FENG $^{1}$ and GUANGXIN LV ${ }^{1}$ \\ ${ }^{1}$ Department of Emergency, The Cangzhou Central Hospital; ${ }^{2}$ Department of The Second Osteology, \\ The Cangzhou Hospital of Integrated Traditional and Western Medicine, Cangzhou, Hebei 061001, P.R. China
}

Received December 9, 2016; Accepted July 20, 2017

DOI: $10.3892 / \mathrm{mmr} .2017 .8010$

\begin{abstract}
The aim of the present study was to explore the roles of human antigen $\mathrm{R}(\mathrm{HuR})$ in sepsis. Reverse transcription-quantitative polymerase chain reaction and western blot analyses demonstrated that overexpression of HuR increased the expression of high-mobility group box 1 (HMGB1) in human umbilical vein endothelial cells (HUVECs). HMGB1 was investigated as a potential target of HuR through bioinformatics and RNA-immunoprecipitation assays. Furthermore, treatment with HuR small interfering (si)RNA suppressed the lipopolysaccharide (LPS)-mediated release of HMGB1 and reduced HMGB1-mediated hyperpermeability and leukocyte migration in HUVECs and in septic mice. In addition, HuR-siRNA injection reduced cecal ligation and puncture (CLP)-induced HMGB1 release, reduced production of interleukin 6 and lowered mortality rates. Notably, the promotive effects of HuR overexpression on the inflammatory response were attenuated when HUVECs were co-treated with HMGB1 short hairpin RNA. Therefore, the present results indicated that the ectopic expression of HuR may induce inflammatory responses and thus sepsis by activating the HMGB1 signaling pathway.
\end{abstract}

\section{Introduction}

Sepsis is a systemic and overwhelming lethal syndrome following local infection (1); however, therapeutic choices are limited for sepsis. Therefore, it is important to elucidate the mechanisms that mediate the development of sepsis. The pathogenesis of sepsis is attributed to pro-inflammatory cytokines that cause inflammation in tissues. Thus, the targeting of late proinflammatory mediators may be an ideal therapy for sepsis. High-mobility group box 1 (HMGB1) is a late

Correspondence to: Dr Jian Wang, Department of Emergency, The Cangzhou Central Hospital, 16 Xinhua Road, Cangzhou, Hebei 061001, P.R. China

E-mail: jianwang_czh@163.com

Key words: human antigen $\mathrm{R}$, high-mobility group box 1 , inflammation, lipopolysaccharide, sepsis mediator of lethal endotoxemia and circulating HMGB1 level is elevated in a delayed fashion in septic mice (2). HMGB1 has previously been reported to disturb the barrier integrity of endothelial cells and induce sepsis by promoting proinflammatory cytokines secretion $(3,4)$. Therefore, HMGB1 may provide an opportunity for clinical intervention for sepsis, which suggested a need to investigate the mechanisms by which HMGB1 is modulated during sepsis.

Post-transcriptional regulation is a crucial event, which includes regulating mRNA stability in inflammatory processes (5). Human antigen $\mathrm{R}(\mathrm{HuR})$ is an important RNA-binding protein; its levels of expression have been reported to be increased in various tumors $(6,7)$, which indicated that HuR may exert a promotive role in tumor development. However, whether HuR is involved in sepsis and the related mechanisms remain unclear.

The present study used bioinformatics analyses to demonstrate that HuR may bind to HMGB1. Reverse transcription-quantitative polymerase chain reaction (RT-qPCR) and western blot analyses revealed that ectopic expression of HuR led to increased HMGB1 expression. Luciferase reporter and co-immunoprecipitation assays demonstrated that HuR was able to bind to HMGB1 directly and thus enhanced the stability of HMGB1 mRNA. In addition, treatment with HuR small interfering (si)RNA suppressed the lipopolysaccharide (LPS)-mediated HMGB1 release and attenuated HMGB1-mediated hyperpermeability and leukocyte migration in vitro and in vivo. Injection of HuR-siRNA also downregulated cecal ligation and puncture (CLP)-induced HMGB1 release, reduced the production of interleukin (IL)-6 and lowered mortality rates. Finally, the promotive effects of HuR overexpression on the inflammatory response were attenuated when human umbilical vein endothelial cells (HUVECs) were co-treated with HMGB1 short hairpin (sh)RNA. These results suggested that HuR may be able to directly bind to and regulate HMGB1 expression during sepsis.

\section{Materials and methods}

Cell culture. HUVECs were obtained from American Type Culture Collection (Manassas, VA, USA) and cultured in Dulbecco's Modified Eagle's Medium (Gibco; Thermo Fisher Scientific, Inc., Waltham, MA, USA) supplemented with $10 \%$ 
heat-inactivated fetal bovine serum (Gibco; Thermo Fisher Scientific, Inc.), $80 \mathrm{U} / \mathrm{ml}$ penicillin, $0.08 \mathrm{mg} / \mathrm{ml}$ streptomycin and $2 \mathrm{mM}$ glutamine at $37^{\circ} \mathrm{C}$ under humidified atmosphere with $5 \% \mathrm{CO}_{2}$.

Animal model of sepsis. All animal experiments were performed with the approval of the Cangzhou Central Hospital Ethics Committee for Animal Experimentation (Cangzhou, China). A total of 60 male C57BL/6 mice (age, 8 weeks; weight, 20-25 g) were purchased from the Medical Center of Yangzhou University (Yangzhou, China). Mice were housed and fed at $27^{\circ} \mathrm{C}$, and 12-h light/dark cycle and had access to food/water. Sepsis was induced by CLP, following a previously published protocol (8). HuR-siRNA (10 nM; Biomics Biotechnology Inc., Nantong, China; Table I) was injected in the tail vein 3 days post-sepsis to detect the effects of HuR on CLP-induced HMGB1 release, IL-6 production and mortality, a scrambled-siRNA was used as a control, following the manufacture's siRNA injection procedure.

ELISA. ELISA kits of HMGB1 (cat. no. H6-NBP1-42888), interleukin (IL)-6 (cat. no. NBP1-92668), tumor necrosis factor (TNF)- $\alpha$ (cat. no. NBP2-31085), IL-1 $\beta$ (cat. no. KA0357) and IL-10 (cat. no. KA1038) were purchased from Novus Biologicals, LLC, Littleton, CO, USA). Toll-like receptor (TLR)2 (cat. no. EK1130) ELISA kit was purchased from Wuhan Boster Biological Technology, Ltd., (Wuhan, China) TLR4 (cat. no. kt56334) and nuclear factor (NF)- $\kappa \mathrm{B}$ (cat. no. kt21004) ELISA kits were purchased from MSK (Wuhan, China), which were used to detect the cytokines level in septic mice serum according to the manufacture's protocols.

Adenovirus (Ad) vector construction. OBiO Technology Corp., Ltd. (Shanghai, China) constructed the HuR overexpression (Ad-HuR), knockdown (Ad-HuR-shRNA; Table I) and HMGB1 overexpression (Ad-HMGB1) adenovirus vectors, which were verified by DNA sequencing by SprinGenBioTech (Nanjing, China).

Reverse transcription-quantitative polymerase chain reaction (RT-qPCR). Total RNA was extracted from $1 \times 10^{6}$ HUVECs with TRIzol reagent (Invitrogen; Thermo Fisher Scientific, Inc.), following the manufacturer's protocol. First-strand cDNA was synthesized from the total RNA using a QuantiTect Rev. Transcription kit (cat. no. 205311; Qiagen China Co., Ltd., Shanghai, China). qRT-PCR was performed on the StepOnePlus PCR system with SYBR Green kit (Biomics Biotechnologies Co., Ltd., Nantong, China). $\beta$-actin was used as an internal control and gene expression levels were normalized to $\beta$-actin, and qRT-PCR reactions were performed at $58^{\circ} \mathrm{C}$ for 35 cycles in triplicate for each experiment. Primer sequences are listed in Table I. The relative gene expression levels were analyzed via $2^{-\Delta \Delta \mathrm{Cq}}$ method (9).

$m R N A$ stability assays. HuR expression level was upregulated or downregulated by infecting with Ad-HuR or Ad-HuR-shRNA at $1 \times 10^{6}$ multiplicity of infection and 70-80\% of cell density, respectively, for at $37^{\circ} \mathrm{C}$ with DMEM medium for $48 \mathrm{~h}$. Subsequently, de novo RNA synthesis was blocked with the addition of $5 \mu \mathrm{g} / \mathrm{ml}$ actinomycin D (ActD; Apexbio,
Houston, TX USA) to the medium. Total RNA was harvested at the $0,2,4,6,8$ and $10 \mathrm{~h}$ post-transfection, and HMGB1 mRNA expression levels were measured by RT-qPCR assay. mRNA half-life was examined by comparing with the mRNA expression level prior to the addition of ActD.

RNA immunoprecipitation (RIP) assays. RIP assays were conducted as previously described (10). Briefly, HUVECs $\left(1 \times 10^{7}\right)$ were lysed with $25 \mathrm{mM}$ Tris- $\mathrm{HCl}$ buffer $(\mathrm{pH} 7.5)$ and $100 \mathrm{U} / \mathrm{ml}$ RNase inhibitor (Sigma-Aldrich; Merck KGaA, Darmstadt, Germany), and subsequently incubated with protein-A sepharose beads (P001, 7Sea Biotech, Shanghai, China) precoated with $3 \mu \mathrm{g}$ anti-HuR antibody or control rabbit immunoglobulin $\mathrm{G}$ for $1.5 \mathrm{~h}$ at $4^{\circ} \mathrm{C}$. The RNA-protein complexes were pulled-down by protein $\mathrm{A} / \mathrm{G}$ agarose beads, RNA was extracted with TRIzol and HMGB1 expression levels were detected with RT-qPCR.

Luciferase reporter assays. The HMGB1 promoter sequence was inserted into the pGL3 vector (pGL3-HMGB1; Promega Corporation, Madison, WI, USA), which was transfected in HUVECs with Ad-HuR or Ad-HuR-shRNA co-infection. Transfection efficiency was normalized with $\beta$-gal activity, and the luciferase activity of pGL3-HMGB1 was measured for HMGB1 promoter transcriptional activity assays. Cells at a density of $80 \%$ were used for transfection with Lipofectamine ${ }^{\circledR} 2000$ (Thermo Fisher Scientific, Inc) at $37^{\circ} \mathrm{C}$ with DMEM medium for $72 \mathrm{~h}$. The luciferase activity was measured by a Glomax 96 luminometer (Promega Corporation).

Cell viability assays. Cell Counting Kit-8 (CCK-8, Vazyme Biotech Co., Ltd., Nanjing, China) was used to examine cell viability. Cells infected with Ad-HuR-shRNA or Ad-empty vector were seeded $(4,000$ cells/well) in 96-well plates and incubated for 24,48 and $72 \mathrm{~h}$ at $37^{\circ} \mathrm{C}$; growth rates were determined by measuring optical density with a microplate reader. Each experiment was performed in triplicate.

In vitro permeability assay. HUVECs were seeded (8x10 4 cells/well) onto Transwell inserts (Millipore; Merck $\mathrm{KGaA}$ ) for $6 \mathrm{~h}$ and subsequently treated with HMGB1 cytokine ( $1 \mu \mathrm{g} / \mathrm{ml}$; cat.no. 34-8401-82; Thermo Fisher Scientific, Inc.) for $16 \mathrm{~h}$ followed by infection with or without Ad-HuR-shRNA for $24 \mathrm{~h}$. Transwell inserts were then washed with PBS, followed by addition of $0.5 \mathrm{ml}$ of Evans blue $(0.67 \mathrm{mg} / \mathrm{ml})$ diluted in growth medium containing $5 \%$ bovine serum albumin (BSA). Fresh growth medium was then added to the lower chamber, and the medium in the upper chamber was replaced with Evans blue/BSA. Following $10 \mathrm{~min}$ incubation, optical density was measured at $650 \mathrm{~nm}$ in the lower chamber.

In vivo permeability and leukocyte migration assays. CLP-operated 12 mice or control 12 mice were pretreated with HMGB1 $(2 \mu \mathrm{g})$ intravenously for $16 \mathrm{~h}$, after which Ad-HuR-siRNA was injected intravenously. A total of 12 mice treated with a scrambled siRNA were used as a control group. After 48 h, 1\% Evans blue dye solution in normal saline was injected intravenously into each mouse, and the mice were sacrificed $30 \mathrm{~min}$ post-injection. The peritoneal exudates 
Table I. Primer sequences used for reverse transcription-quantitative polymerase chain reaction, siRNA and shRNA.

\begin{tabular}{ll}
\hline Gene & \multicolumn{1}{c}{ Sequences $\left(5^{\prime} \rightarrow 3^{\prime}\right)$} \\
\hline HMGB1 & F: CTTCCTCTTCTGCTCTGAGTATCG \\
& R: CTTCCTTCTTTTCTTGCTTTTT \\
E-selectin & F: AACTGCGAGAAGAACGGATAGAGA \\
& R: AGCGAGGAGAACAAAAACAAGAGC \\
HuR & F: CTGGTGTCCAAAAGTCAACAATAA \\
& R: AAAAAAAAAAATAAAAAGGCAATG \\
$\beta$-actin & F: ATCCACGAAACTACCTTCAACTCC \\
& R: GATCTTGATCTTCATTGTGCTGGG \\
ICAM-1 & F: GATTGTCATCATCACTGTGGTAGC \\
& R: GGCCTGTTGTAGTCTGTATTTCTT \\
VCAM-1 & F: ATACAACCGTCTTGGTCAGCCCTT \\
& R: CATTCATATACTCCCGCATCCTTC \\
HuR siRNA & GAGGCAAUUACCAGUUUCA \\
& F: CCGGTGAGGCAATTACCAGTTCACTC \\
& GAGTGAAACTGGTAATTGCCTCTTTTG \\
& R: AATTCAAAAAAGGCAATTACCAGTTT \\
& CACTCGAGTGAAACTGGTAATGCCTCA
\end{tabular}

F, forward; HMGB1, high-mobility group box 1; HuR, human antigen R; ICAM, intercellular adhesion molecule; R, reverse; sh, short hairpin; si, small interfering; VCAM, vascular cell adhesion molecule.

were collected following washes with $5 \mathrm{ml}$ normal saline and centrifuged at $200 \mathrm{xg}$ for $10 \mathrm{~min}$ at $4^{\circ} \mathrm{C}$. The absorbance of the supernatant was read at $650 \mathrm{~nm}$ via a 96 -well plate by GloMax ${ }^{\circledR}$ Discover System (GM3000; Promega Corporation). Vascular permeability was expressed in terms of dye $(\mu \mathrm{g}) /$ mouse. To assess leukocyte and neutrophil migration, $20 \mu \mathrm{l}$ of peritoneal fluid was mixed with $0.38 \mathrm{ml}$ of Turk's solution $(0.01 \%$ crystal violet in $3 \%$ acetic acid), and the leukocyte number was counted under a light microscope. The results were expressed as neutrophil $\times 10^{6}$ per peritoneal cavity.

Statistical analysis. All data are presented as mean \pm standard deviation. The differences between the groups were analyzed with the Student's t-test or one-way analysis of variance of three independent experiments. The differences between the groups were analyzed using a one-way analysis of variance with the Tukey-Kramer post-hoc test. $\mathrm{P}<0.05$ was considered to indicate a statistically significant difference.

\section{Results}

Overexpression of $H u R$ increases $H M G B 1$ expression in HUVECs. The present study identified that HuR has 21 potential binding sites on HMGB1 in 11 different cancers and the binding ability ranked first by the starBase v2.0 (http://starbase.sysu.edu.cn/browseMrnaCeRNA.php) analysis (Table II). Subsequently, the ability of HuR to increase HMGB1 expression in HUVECs was examined. Transfection with Ad-HuR or Ad-HuR-shRNA resulted in the subsequent increase or decrease, respectively, of HuR mRNA and protein expression levels in HUVECs (Fig. 1A-D). In addition, overexpression of HuR significantly upregulated HMGB1 mRNA and protein expression levels (Fig. 1E and F, respectively). By contrast, HuR-knockdown led to a significant decrease in HMGB1 mRNA and protein expression (Fig. $1 \mathrm{G}$ and $\mathrm{H}$, respectively). These results indicated that HuR was able to positively regulate HMGB1 expression.

HuR may physically interact with HMGBI in HUVECs. Whether HuR was able to regulate HMGB1 mRNA stability in HUVECs was examined. HuR expression was induced or knocked down followed by the inhibition of de novo mRNA synthesis by ActD treatment. The decay rate of HMGB1 mRNA was faster in Ad-HuR-shRNA infected cells $\left(\mathrm{t}_{1 / 2}=3.8 \pm 0.4 \mathrm{~h}\right)$ compared with control cells $\left(\mathrm{t}_{1 / 2}=5.4 \pm 0.3 \mathrm{~h}\right.$; Fig. $\left.2 \mathrm{~A}\right)$, and slower in the Ad-HuR-treated group $\left(\mathrm{t}_{1 / 2}=7.9 \pm 0.2 \mathrm{~h}\right)$ compared with the control cells $\left(t_{1 / 2}=5.3 \pm 0.4 \mathrm{~h}\right.$; Fig. $\left.2 \mathrm{~B}\right)$. However, the luciferase reporter assay demonstrated that the promoter transcriptional activity of HMGB1 was not affected by Ad-HuR or Ad-HuR-shRNA infection in HUVECs (Fig. 2C), which indicate that $\mathrm{HuR}$ could not regulate the transcriptional activity of HMGB1. Furthermore, RIP assays demonstrated that HuR interacted with HMGB1 more specifically than the control group (Fig. 2D). Our results demonstrate that HuR may interact with HMGB1 and enhances HMGB1 mRNA stability in HUVECs.

Effects of HuR on LPS- and CLP-mediated release of HMGB1. Previous studies have shown that LPS induced HMGB1 release in human endothelial cells $(11,12)$. The release of HMGB1 was stimulated with LPS $(100 \mathrm{ng} / \mathrm{ml})$ treatment in HUVECs (Fig. 3A). HUVECs were transfected with Ad-HuR-shRNA and subsequently stimulated with LPS for $24 \mathrm{~h}$. Knockdown of HuR with Ad-HuR-shRNA attenuated the inductive effects of LPS on HMGB1 release (Fig. 3B). However, HUVECs treated with Ad-HuR-shRNA alone did not affect the release of HMGB1 at $24 \mathrm{~h}$, but decreased HMGB1 release at 48 and $72 \mathrm{~h}$ (Fig. 3C). As CLP-induced sepsis in mice closely resembles human sepsis (13), mice were subjected to sepsis by CLP treatment. Notably, injection of HuR-siRNA inhibited the CLP-mediated release of HMGB1 (Fig. 3D).

In addition, the effects of HuR on HMGB1 receptors TLR2 and TLR4 were detected in HUVECs. The results indicated that infection with Ad-HMGB1 significantly induced TLR2 and TLR4 protein expression, which was attenuated by co-infection with Ad-HuR-shRNA (Fig. 3E). To exclude the possibility that the decline of HMGB1 release was due to cytotoxicity caused by Ad-HuR-shRNA infection, cell viability was examined following Ad-HuR-shRNA infection by CCK-8 assay. Ad-HuR-shRNA did not affect cell viability at 24, 48 or $72 \mathrm{~h}$ post-transfection (Fig. 3F). Therefore, these results suggested that HuR-knockdown may attenuate the LPS- and CLP-mediated HMGB1 release.

Effects of HuR-siRNA transfection on LPS or HMGB1mediated barrier disruption. The effects of HuR on the barrier integrity of HUVECs were also examined. No significant difference was identified in barrier integrity in 
Table II. Human RNA-binding protein-mRNA interactions.

\begin{tabular}{|c|c|c|c|c|c|}
\hline Protein & Gene & Target sites & Biocomplex & ClipReadNum & cancerNum \\
\hline HuR & HMGB1 & 21 & 3 & 21 & 11 \\
\hline РТВ & HMGB1 & 3 & 1 & 28 & 9 \\
\hline IGF2BP1 & HMGB1 & 7 & 1 & 141 & 5 \\
\hline IGF2BP2 & HMGB1 & 8 & 1 & 278 & 5 \\
\hline IGF2BP3 & HMGB 1 & 12 & 1 & 310 & 6 \\
\hline TNRC6 & HMGB1 & 1 & 1 & 14 & 9 \\
\hline eIF4AIII & HMGB1 & 18 & 2 & 86 & 11 \\
\hline FMRPH & HMGB1 & 4 & 2 & 4000 & 7 \\
\hline FXR2 & HMGB1 & 2 & 1 & 2000 & 7 \\
\hline FUS & HMGB1 & 17 & 4 & 1052 & 9 \\
\hline LIN28A & HMGB1 & 4 & 1 & 25 & 5 \\
\hline LIN28B & HMGB1 & 3 & 1 & 28 & 3 \\
\hline MOV10 & HMGB1 & 7 & 1 & 7000 & 11 \\
\hline CAPRIN1 & HMGB1 & 2 & 1 & 1350 & 8 \\
\hline EWSR1 & HMGB1 & 1 & 1 & 1000 & 10 \\
\hline
\end{tabular}
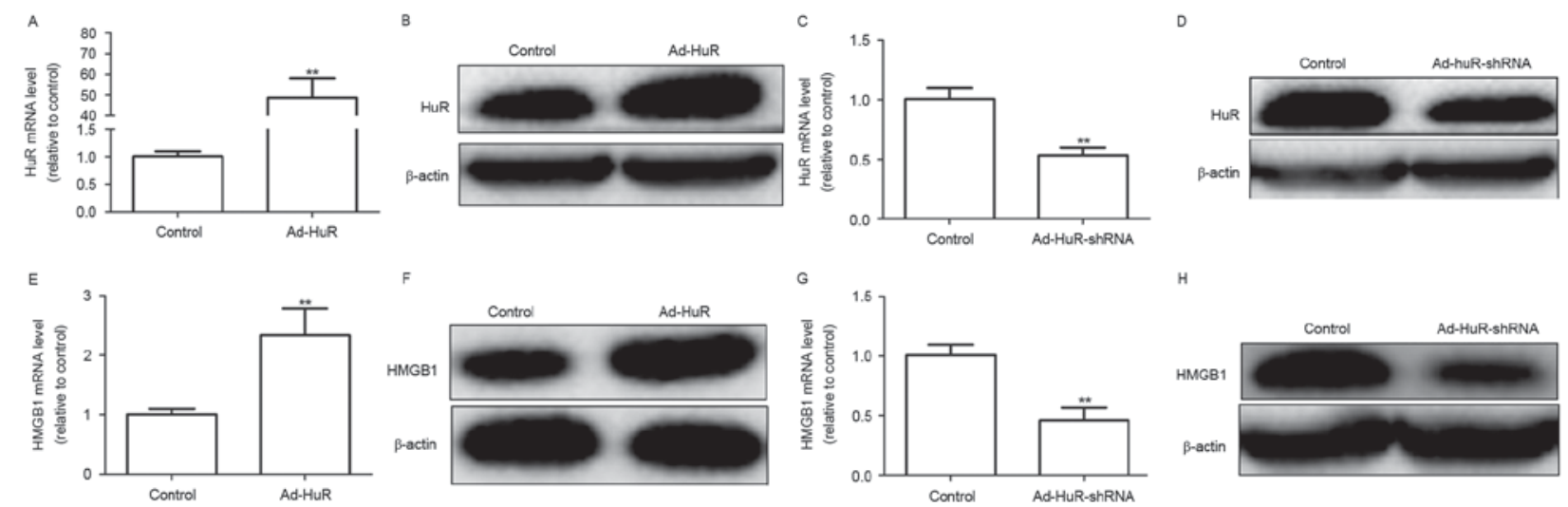

Figure 1. Ectopic expression of HuR promotes HMGB1 expression in HUVECs. (A) HuR mRNA and (B) HuR protein levels were detected in HUVECs infected with Ad-HuR by RT-qPCR and western blot analysis, respectively. (C) RT-qPCR and (D) western blot analyses were applied to examine the mRNA and protein levels, respectively, of HuR in HUVECS transfected with Ad-HuR-shRNA. (E) HMGB1 mRNA and (F) HMGB1 protein levels were detected in HUVECs infected with Ad-HuR by RT-qPCR and western blot assays, respectively. (G) RT-qPCR and (H) western blot analyses were applied to examine the mRNA and protein levels, respectively, of HMGB1 in HUVECs transfected with Ad-HuR-shRNA. Data are presented as the mean \pm standard deviation; ${ }^{* *} \mathrm{P}<0.01$ vs. empty vector control. Ad, adenovirus; HMGB1, high-mobility group box 1 ; HuR, human antigen R; HUVECs, human umbilical vein endothelial cells; RT-qPCR, reverse transcription-quantitative polymerase chain reaction; shRNA, short hairpin RNA.

cells transfected with Ad-HuR-shRNA alone compared with control transfected cells (Fig. 4A). As endothelial membrane barriers were previously demonstrated to be disrupted by LPS induction (14), HUVECs were transfected with Ad-HuR-shRNA for $24 \mathrm{~h}$ following LPS treatment. Knockdown of HuR attenuated the LPS-mediated membrane disruption (Fig. 4B). In addition, the barrier integrity has been reported to be perturbed by HMGB1 induction (15). Therefore, HUVECs were transfected with HMGB1 followed by Ad-HuR-shRNA co-transfection. Cells co-transfected with Ad-HuR-shRNA exhibited an decrease in HMGB1-mediated membrane disruption (Fig. 4C). In addition, these effects were investigated in vivo by examining HMGB1- or CLP-induced vascular permeability in mice. Treatment with HuR-shRNA suppressed HMGB1- or CLP-induced peritoneal leakage of dye (Fig. 4D and E, respectively). These results demonstrated that the knockdown of HuR expression may reduce HMGB1-mediated endothelial disruption and maintain the human endothelial cell barrier integrity.

HuR-knockdown inhibits the expression of cell adhesion molecules (CAMs) and proinflammatory responses. A previous study reported that the inflammatory effects mediated by HMGB1 were caused by increasing the cell surface expression of intracellular adhesion molecule (ICAM)-1, vascular (V)CAM-1 and E-selectin, which promote leukocyte adhesion and migration from the endothelium to inflammation sites and hence of the proinflammatory response (16). Transfection with Ad-HuR-shRNA suppressed the mRNA expression levels of ICAM-1, VCAM-1 and E-selectin (Fig. 5A). The binding ability of leukocytes to HMGB1-stimulated HUVECs was significantly inhibited by HuR knockdown (Fig. 5B). 


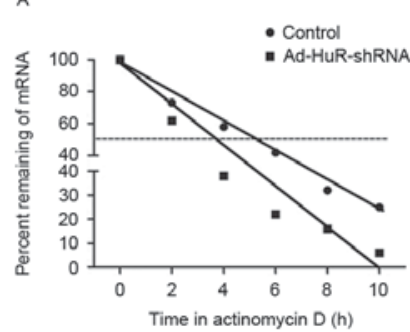

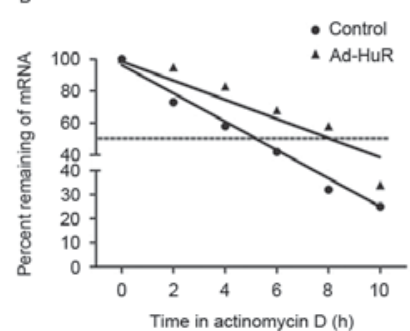

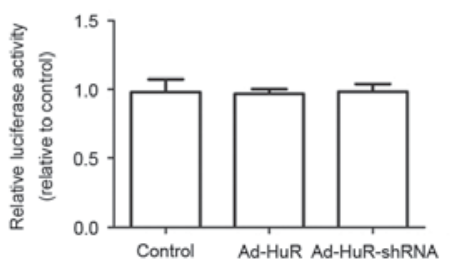

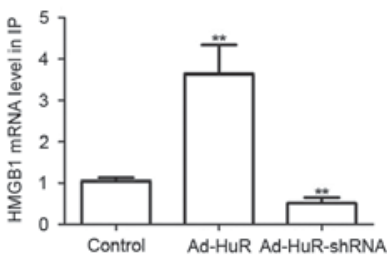

Figure 2. HuR physically interacts with HMGB1 in HUVECs. HUVECs transfected with (A) Ad-HuR-shRNA or (B) Ad-HuR infection were treated with actinomycin D for the indicated times. HMGB1 mRNA levels were measured by RT-qPCR and the percentage of mRNA that remained was plotted. (C) HuR overexpression did not affect the promoter activity of HMGB1. HUVECs transfected with Ad-HuR or Ad-HuR-shRNA were transiently co-transfected with luciferase plasmids containing the promoter sequences of the human HMGB1 gene for $24 \mathrm{~h}$. Luciferase activity in these cells was measured using the Dual-Luciferase Reporter Assay System. (D) RT-qPCR was used to measure the abundance of HMGB1 mRNA present in the HuR-immunoprecipitation materials following the RNA immunoprecipitation assay. Values are presented as the mean \pm standard deviation $(\mathrm{n}=3)$. ${ }^{* *} \mathrm{P}<0.01$ vs. control. Empty vectors were used as control. Ad, adenovirus; HMGB1, high-mobility group box 1; HuR, human antigen R; HUVECs, human umbilical vein endothelial cells; RT-qPCR, reverse transcription-quantitative polymerase chain reaction; shRNA, short hairpin RNA.
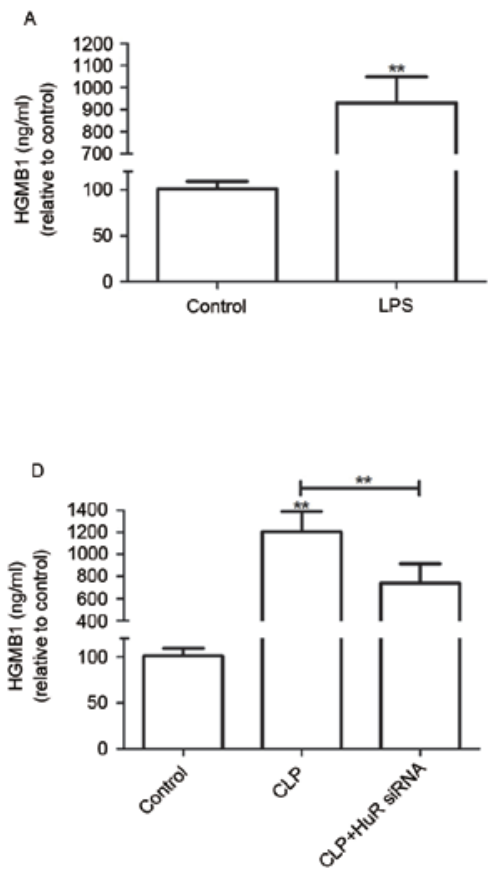

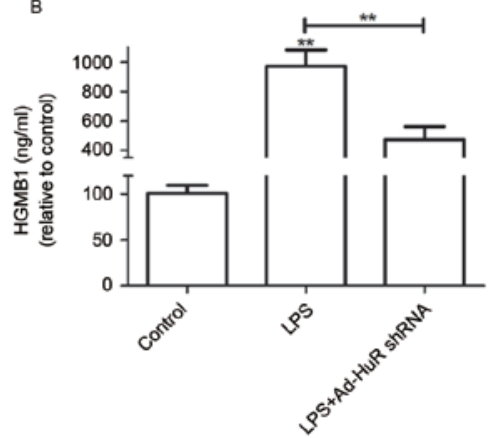

E

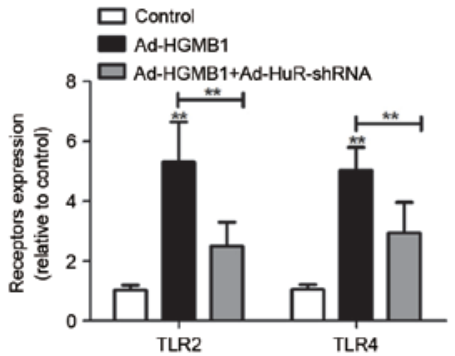

c
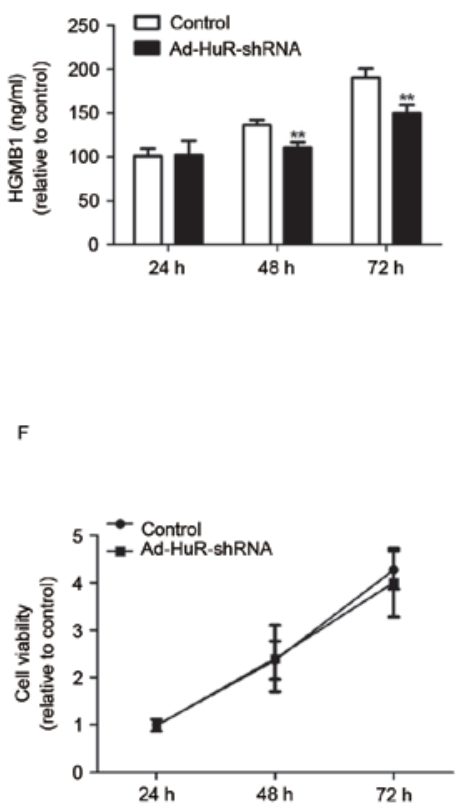

Figure 3. HuR knockdown impairs HMGB1 release and receptor expression. (A) HUVECs were treated with $100 \mathrm{ng} / \mathrm{ml}$ LPS for 16 h, and HMGB1 release was measured by ELISA. (B) HUVECs were transfected with Ad-HuR-shRNA for $24 \mathrm{~h}$ following stimulation with LPS (100 ng/ml) for 16 h; HMGB1 release was examined by ELISA. (C) HMGB1 release was tested by ELISA in Ad-HuR-shRNA-transfected HUVECs for 24, 48 and 72 h. (D) Male C57BL/6 mice underwent CLP surgery and subsequently injected with HuR-siRNA intravenously at $12 \mathrm{~h}$ post-CLP (n=5). Mice were euthanized $24 \mathrm{~h}$ following CLP. Serum HMGB1 levels were measured by ELISA. (E) Confluent HUVECs were incubated with Ad-HMGB1 (1 $\mu \mathrm{g} / \mathrm{ml})$ for $16 \mathrm{~h}$; cells were subsequently infected with or without Ad-HuR-shRNA for 24 h. Expression levels of TLR2 and TLR4 in HUVECs were measured by cell-based ELISA. (F) The effects of Ad-HuR-shRNA on cellular viability were measured using the Cell Counting Kit- 8 assay. Values are presented as the mean \pm standard deviation; $\mathrm{n}=3$; ${ }^{* *} \mathrm{P}<0.01$. Empty vectors and Scrambled-siRNA were used as controls. Ad, adenovirus; CLP, cecal ligation and puncture; HMGB1, high-mobility group box 1; HuR, human antigen R; HUVECs, human umbilical vein endothelial cells; LPS, lipopolysaccharide; shRNA, short hairpin RNA; siRNA, small interfering RNA; TLR, toll-like receptor.

In addition, HuR-knockdown inhibited the association of leukocytes to HUVEC binding with the subsequent transendothelial migration of leukocytes (Fig. 5C). Notably, mice injected with HuR-siRNA exhibited an inhibition of HMGB1or CLP-induced migration of neutrophils (Fig. 5D and E). The results demonstrated that knockdown of HuR inhibits HMGB1-induced proinflammatory signaling and thereby downregulates the activation of inflammatory pathways, such as the upregulation of CAMs, leukocyte adhesion and migration induced by HMGB1.
HuR-knockdown inhibits HMGB1-stimulated production of TNF- $\alpha$ and $I L-1 \beta$. To investigate the potential effects of HuR-knockdown on the induction of inflammatory signaling molecules, the production levels of TNF- $\alpha$ and IL-1 $\beta$ were detected in HMGB1-activated HUVECs. The production of TNF- $\alpha$ and IL-1 $\beta$ was increased by HMGB1 treatment (Fig. 6A and B, respectively), which was attenuated by HuR knockdown with Ad-HuR-shRNA. In addition, the effects of HuR knockdown on the $\mathrm{NF}-\kappa \mathrm{B}$ pathway were evaluated. Knockdown of HuR inhibited the promotive effects of 

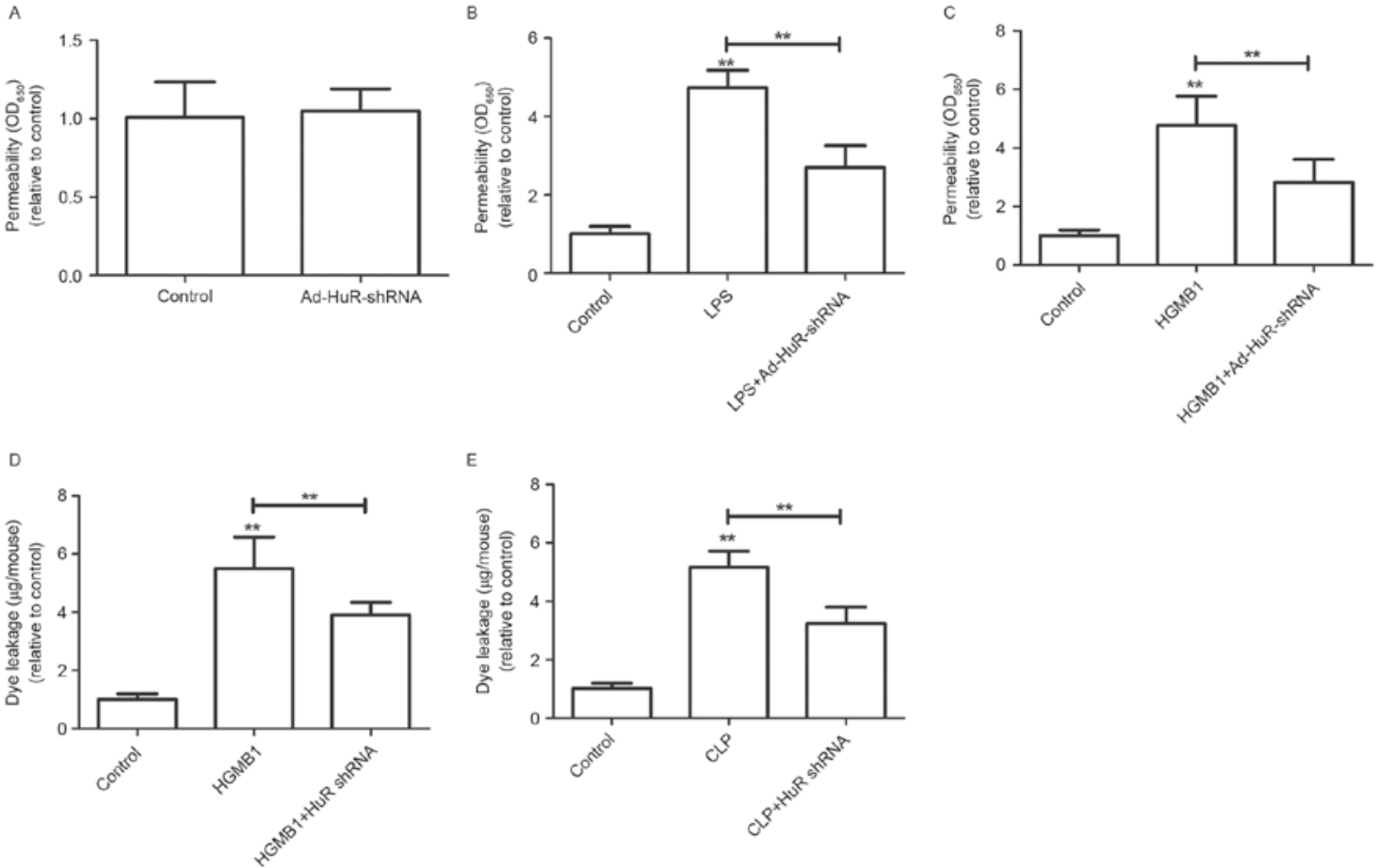

Figure 4. Ad-HuR-shRNA impairs HMGB1-mediated permeability in vitro and in vivo. (A) HUVECs were infected with Ad-HuR-shRNA for 24 h, and permeability was monitored by measuring the flux of Evans blue-bound albumin across HUVECs ( $\mathrm{n}=3$ ). HUVECs were stimulated with (B) LPS (100 ng/ml for $4 \mathrm{~h}$ ) or (C) HMGB1 $(1 \mu \mathrm{g} / \mathrm{ml}, 16 \mathrm{~h})$ and subsequently infected with Ad-HuR-shRNA for $24 \mathrm{~h}$. Permeability was monitored by measuring the flux of Evans blue-bound albumin across HUVECs $(\mathrm{n}=3)$. The effects of HuR-siRNA injection on (D) HMGB1-induced ( $2 \mu \mathrm{g} / \mathrm{mouse})$ or (E) CLP-induced (24 h post-CLP) vascular permeability in mice were examined by measuring the amount of Evans blue in peritoneal washings ( $\mathrm{n}=5$ ). Values are presented as the mean \pm standard deviation; ${ }^{* *} \mathrm{P}<0.01$ vs. control. Empty vector and Scrambled-siRNA were used as controls. Ad, adenovirus; CLP, cecal ligation and puncture; HMGB1, high-mobility group box 1; HuR, human antigen R; HUVECs, human umbilical vein endothelial cells; LPS, lipopolysaccharide; shRNA, short hairpin RNA; siRNA, small interfering RNA.
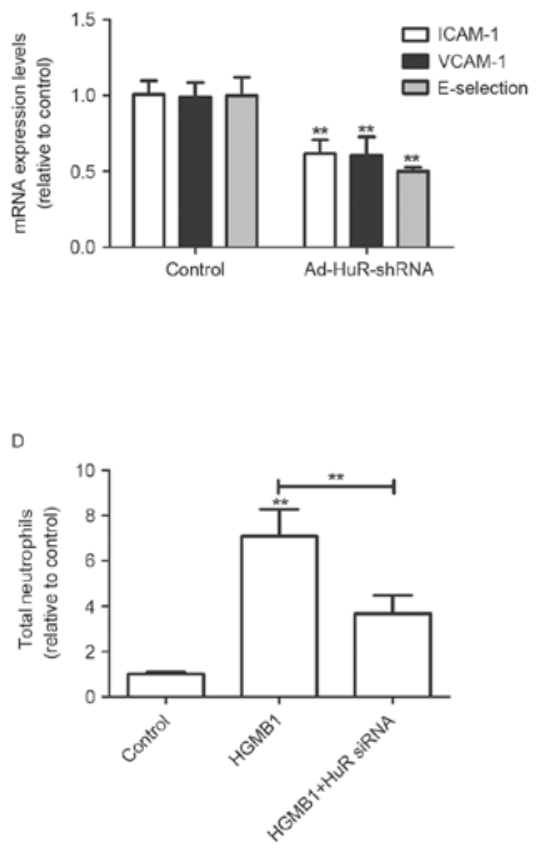
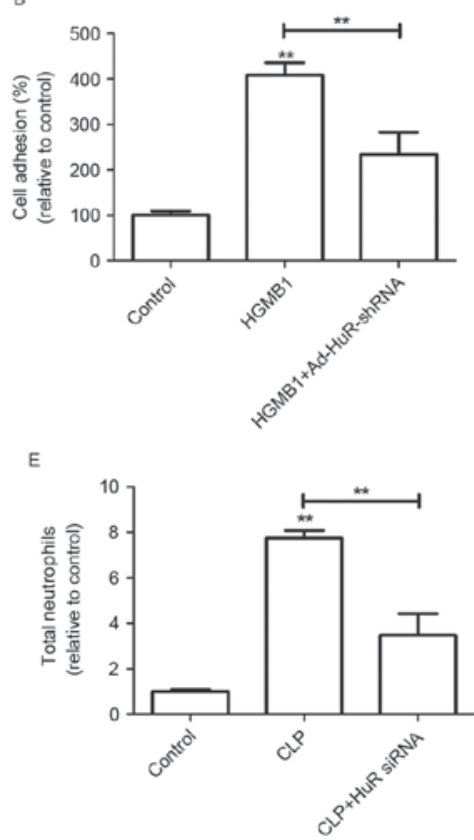

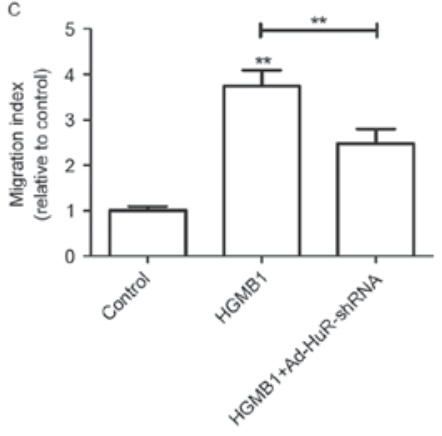

Figure 5. Effects of HuR knockdown on HMGB1-mediated pro-inflammatory responses. (A) HUVECs were stimulated with HMGB1 (1 $\mu \mathrm{g} / \mathrm{ml})$ for $16 \mathrm{~h}$ followed by transfection with Ad-HuR-shRNA. HMGB1-mediated expression levels of VCAM-1, ICAM-1 and E-selectin in HUVECs were detected by reverse transcription-quantitative polymerase chain reaction. (B) Adherence of neutrophils to HUVECs and (C) migration of monocytes through HUVECs were analyzed. Male C57BL/6 mice (D) were stimulated with HMGB1 or (E) received CLP surgery and were subsequently co-treated with HuR-siRNA, and HMGB1- or CLP-mediated migration of leukocytes into the peritoneal cavity of mice was analyzed. Values are presented as the mean \pm standard deviation; $n=3 ;{ }^{* *} \mathrm{P}<0.01$ vs. control. Empty vectors and Scramble-siRNA were used as a controls. Ad, adenovirus; CLP, cecal ligation and puncture; HMGB1, high-mobility group box 1; HuR, human antigen R; HUVECs, human umbilical vein endothelial cells; ICAM, intercellular adhesion molecule; shRNA, short hairpin RNA; siRNA, small interfering RNA; VCAM, vascular cell adhesion molecule. 

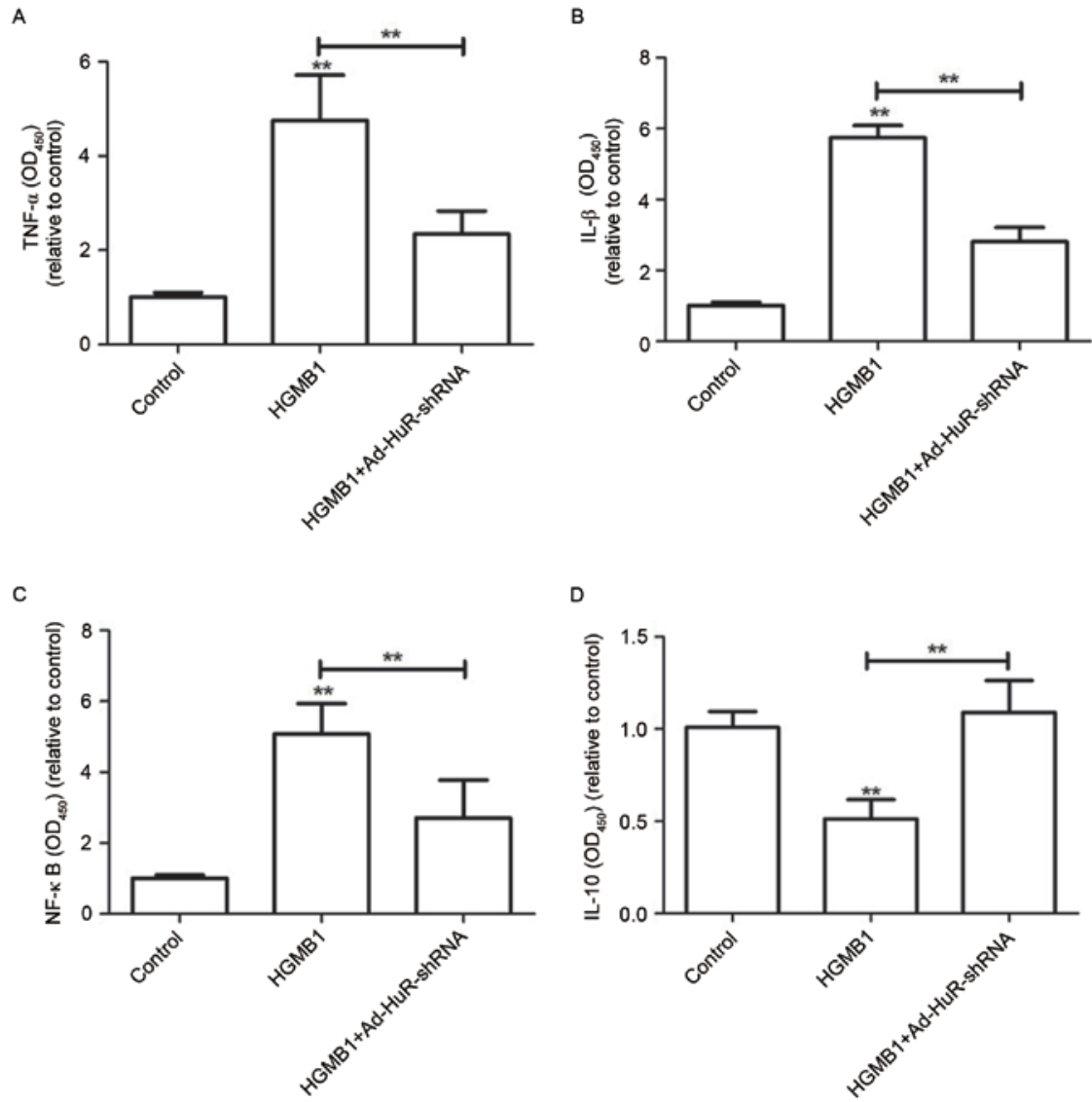

Figure 6. Effects of HuR knockdown on HMGB1-stimulated activation of NF- $\kappa B$ and production of TNF- $\alpha$ and IL-1 $\beta$ in HUVECs. HUVECs were stimulated with HMGB1 $(1 \mu \mathrm{g} / \mathrm{ml})$ for $16 \mathrm{~h}$, followed by transfection with or without Ad-HuR-shRNA for $24 \mathrm{~h}$. HMGB1-mediated production of (A) TNF- $\alpha$, (B) IL-1 $\beta$ (C) total NF- $\mathrm{B}$ (D) IL-10 in HUVECs were analyzed. Values are presented as the mean \pm standard deviation; $\mathrm{n}=3$; ${ }^{* *} \mathrm{P} 0.01$ vs. control. Empty vectors were used as control. Ad, adenovirus; HMGB1, high-mobility group box 1; HuR, human antigen R; HUVECs, human umbilical vein endothelial cells; IL, interleukin; NF- $\mathrm{kB}$, nuclear factor $\kappa \mathrm{B}$; shRNA, short hairpin RNA; TNF, tumor necrosis factor.

A

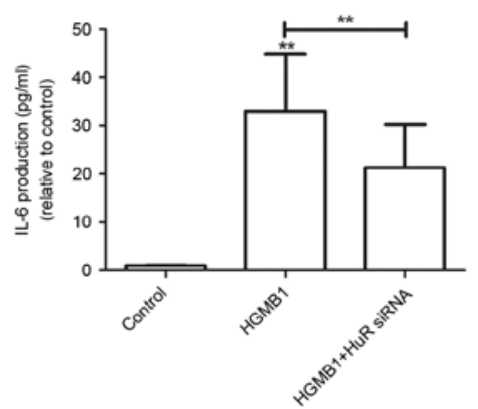

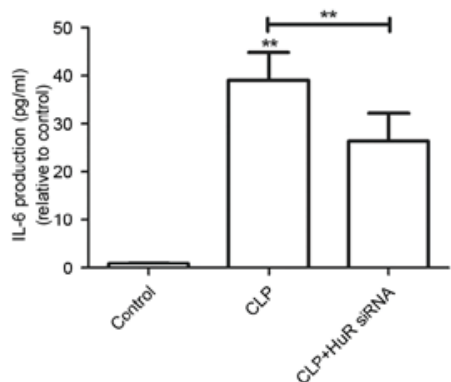

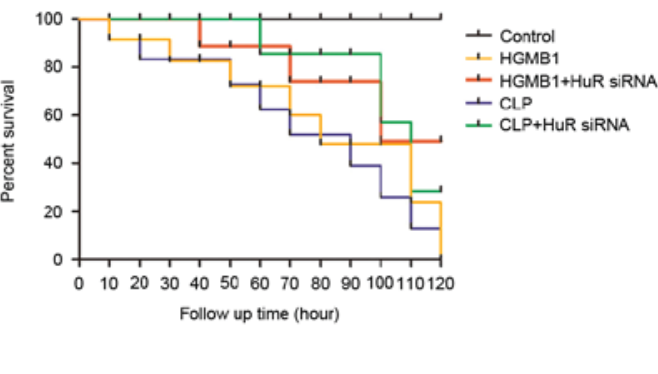

Figure 7. Effects of HuR-siRNA treatment on the production of IL-6 and survival rates following HMGB1 or CLP treatment. Male C57BL/6 mice underwent (A) HMGB1 treatment or (B) CLP surgery and were subsequently injected with HuR-siRNA intravenously $12 \mathrm{~h}$ post-CLP or HMGB1 treatment ( $\mathrm{n}=5$ ). Mice were euthanized $96 \mathrm{~h}$ after CLP or HMGB1 treatment. Serum IL-6 levels were measured by ELISA. (C) Male C57BL/6 mice (n=12) were injected with HuR-siRNA at 12 and $60 \mathrm{~h}$ after CLP or HMGB1 treatment, 12 mice treated with a scrambled siRNA were used as a control group. Animal survival was monitored every $10 \mathrm{~h}$ after CLP for $120 \mathrm{~h}$. Control CLP mice were administered sterile saline (n=12). A Kaplan-Meier survival analysis was used to determine overall survival rates vs. CLP-treated mice or HMGB1-treated mice. Values are presented as the mean \pm standard deviation; $\mathrm{n}=3$; ${ }^{* *} \mathrm{P}<0.01$ vs. control. Scramble-siRNA was used as a control. Ad, adenovirus; CLP, cecal ligation and puncture; HMGB1, high-mobility group box 1; HuR, human antigen R; IL, interleukin; siRNA, small interfering RNA.

HMGB1-mediated NF- $\kappa$ B activity (Fig. 6C). IL-10 is an anti-inflammatory cytokine (17); the production of IL-10 was detected in HMGB1-activated HUVECs. The production of IL-10 was decreased by HMGB1 treatment (Fig. 6D), and this effect was reversed by HuR knockdown. These results indicated that HuR may regulate the NF- $\mathrm{KB}$ pathway that is involved in the induction of proinflammatory responses in human endothelial cells.

Protective effects of HuR-siRNA in CLP-induced production of IL-6 and septic mortality. Sepsis has a poor prognosis, which is often characterized as the production of IL-6 $(18,19)$. 
Therefore, the present study hypothesized that treatment with HuR-siRNA would be able to reduce the production of IL-6 and mortality rates in HMGB1- or CLP-induced sepsis mouse model. Production of IL-6 in HMGB1- or CLP-induced mice was reduced by HuR-siRNA injection (Fig. 7A and B, respectively). In addition, HuR-siRNA injection resulted in an increase in the survival rate, analyzed by Kaplan-Meier survival plot (Fig. 7C), which suggests that HuR might be a potential therapeutic target for sepsis.

\section{Discussion}

Survivors of sepsis may exhibit significant cognitive and physical impairments (20). Therefore, it is important to investigate the mechanisms underlying sepsis. Experimentally, sepsis is routinely induced by a number of techniques, such as the infusion of exogenous bacterial toxins and CLP-induced disruption of host epithelial barrier (3). Bacterial toxin-induced models have been previously used to investigate the roles of cytokines in systemic inflammation; however, the CLP-induced model is considered the most clinically relevant experimental model for human sepsis (21). In the present study, CLP-induced model rats were used. The results demonstrated that the knockdown of HuR may attenuate LPS- and CLP-mediated HMGB1 release, and reduce HMGB1-mediated endothelial disruption and maintain the human endothelial cell barrier integrity. Notably, data from the present study indicated that HuR knockdown provided protective effects on CLP-induced production of IL-6 and mortality due to sepsis. A previous study demonstrated that high plasma concentrations of HMGB1 during sepsis may be related to poor prognosis and high mortality (22); the prevention of LPS- or CLP-induced release of HMGB1 by knockdown of HuR in the present study suggested that HuR may be a potential therapeutic target for the treatment of vascular inflammatory diseases.

In mouse models of endotoxemia and CLP-mediated sepsis, HMGB1 was first detected in the circulation $8 \mathrm{~h}$ following onset of the disease, and subsequently increased to plateau levels from 16 to $32 \mathrm{~h}$ (21). However, whether HMGB1 is regulated and the detailed mechanism was unknown. Post-transcriptional regulation, particularly mRNA stability alterations, may lead to the ectopic expression of inflammation-related genes (5). The present study demonstrated that HMGB1 may be a target of HuR in HUVECs. To the best of the authors' knowledge, the present study was the first to reveal a novel mechanism of HuR-mediated regulation HMGB1 in HUVECs.

In conclusion, HuR/HMGB1 may be a potential therapeutic target and provides a strong rationale for the development of HMGB1-based therapeutic strategies for sepsis.

\section{Acknowledgements}

The authors thank Professor $\mathrm{Hu}$ for critical reading of this study.

\section{References}

1. Riedemann NC, Guo RF and Ward PA: Novel strategies for the treatment of sepsis. Nat Med 9: 517-524, 2003.

2. Wang H, Bloom O, Zhang M, Vishnubhakat JM, Ombrellino M, Che J, Frazier A, Yang H, Ivanova S, Borovikova L, et al: HMG-1 as a late mediator of endotoxin lethality in mice. Science 285: 248-251, 1999.

3. Wang H, Ward MF and Sama AE: Targeting HMGB1 in the treatment of sepsis. Expert Opin Ther Targets 18: 257-268, 2014.

4. Huang W, Tang Y and Li L: HMGB1, a potent proinflammatory cytokine in sepsis. Cytokine 51: 119-126, 2010.

5. Gerstberger S, Hafner M and Tuschl T: A census of human RNA-binding proteins. Nat Rev Genet 15: 829-845, 2014.

6. Zhu H, Berkova Z, Mathur R, Sehgal L, Khashab T, Tao RH, Ao X, Feng L, Sabichi AL, Blechacz B, et al: HuR suppresses fas expression and correlates with patient outcome in liver cancer. Mol Cancer Res 13: 809-818, 2015.

7. Poria DK, Guha A, Nandi I and Ray PS: RNA-binding protein HuR sequesters microRNA-21 to prevent translation repression of proinflammatory tumor suppressor gene programmed cell death 4. Oncogene 35: 1703-1715, 2016.

8. Yang M, Cao L, Xie M, Yu Y, Kang R, Yang L, Zhao M and Tang D: Chloroquine inhibits HMGB1 inflammatory signaling and protects mice from lethal sepsis. Biochem Pharmacol 86: 410-418, 2013.

9. Livak KJ and Schmittgen TD: Analysis of relative gene expression data using real-time quantitative PCR and the 2(-Delta Delta C(T)) method. Methods 25: 402-408, 2001.

10. Nabors LB, Gillespie GY, Harkins L and King PH: HuR, a RNA stability factor, is expressed in malignant brain tumors and binds to adenine- and uridine-rich elements within the $3^{\prime}$ untranslated regions of cytokine and angiogenic factor mRNAs. Cancer Res 61: 2154-2161, 2001.

11. Bae JS and Rezaie AR: Activated protein C inhibits high mobility group box 1 signaling in endothelial cells. Blood 118: 3952-3959, 2011.

12. Bae JS and Rezaie AR: Thrombin inhibits HMGB1-mediated proinflammatory signaling responses when endothelial protein $\mathrm{C}$ receptor is occupied by its natural ligand. BMB Rep 46: 544-559, 2013.

13. Buras JA, Holzmann B and Sitkovsky M: Animal models of sepsis: Setting the stage. Nat Rev Drug Discov 4: 854-865, 2005.

14. Berman RS, Frew JD and Martin W: Endotoxin-induced arterial endothelial barrier dysfunction assessed by an in vitro model. $\mathrm{Br}$ J Pharmacol 110: 1282-1284, 1993.

15. Wolfson RK, Chiang ET and Garcia JG: HMGB1 induces human lung endothelial cell cytoskeletal rearrangement and barrier disruption. Microvasc Res 81: 189-197, 2011.

16. Hansson GK and Libby P: The immune response in atherosclerosis: A double-edged sword. Nat Rev Immunol 6: 508-519, 2006.

17. Dagdeviren S, Jung DY and Friedline RH: IL-10 prevents aging-associated inflammation and insulin resistance in skeletal muscle. FASEB J 31: 701-710, 2017.

18. Cohen J: The immunopathogenesis of sepsis. Nature 420: 885-891, 2002.

19. Teiten MH, Eifes S, Dicato M and Diederich M: Curcumin-the paradigm of a multi-target natural compound with applications in cancer prevention and treatment. Toxins 2: 128-162, 2010.

20. Perl TM, Dvorak L, Hwang T and Wenzel RP: Long-term survival and function after suspected Gram-negative sepsis. JAMA 274: 338-345, 1995.

21. Li W, Zhu S, Zhang Y, Li J, Sama AE, Wang P and Wang H: Use of animal model of sepsis to evaluate novel herbal therapies. J Vis Exp pii: 3926, 2012.

22. Yoo $\mathrm{H}, \mathrm{Ku}$ SK, Lee $\mathrm{T}$ and Bae JS: Orientin inhibits HMGB1-induced inflammatory responses in HUVECs and in murine polymicrobial sepsis. Inflammation 37: 1705-1717, 2014. 